\title{
Nonlinear Dynamics of a Bose Condensed Gas
}

\author{
F. Dalfovo, C. Minniti, S. Stringari \\ Dipartimento di Fisica, Università di Trento, and \\ Istituto Nazionale Fisica della Materia, I-38050 Povo, Italy \\ L. Pitaevskii \\ Department of Physics, TECHNION, Haifa 32000, Israel, and \\ Kapitza Institute for Physical Problems, ul. Kosygina 2, 117334 Moscow
}

(December 18, 1996)

\begin{abstract}
We investigate the dynamic behavior of a Bose-condensed gas of alkali atoms interacting with repulsive forces and confined in a magnetic trap at zero temperature. Using the Thomas-Fermi approximation, we rewrite the Gross-Pitaevskii equation in the form of the hydrodynamic equations of superfluids. We present solutions describing large amplitude oscillations of the atomic cloud as well as the expansion of the gas after switching off the trap. We compare our theoretical predictions with the recent experimental data obtained at Jila and MIT.
\end{abstract}

PACS numbers: 3.75.Fi, 67.40.Db, 67.90.+z

The dynamic behavior of Bose-condensed gases of alkali atoms in magnetic traps has been the object of recent experiments at Jila [1,2,3] and MIT [4, 国,6]. Absorption images of the atomic cloud provide quantitative information on the dynamics of the expansion after switching off the trap as well as accurate data for the frequencies of the collective excitations. The experimental results reveal that the role played by the interatomic forces is important in these systems and can not be ignored for a quantitative understanding of the data. This opens new challenging tasks for theoretical investigation.

The natural theory to investigate the dynamic behavior of a nonuniform Bose condensate, at $T=0$, is given by the time-dependent Gross-Pitaevskii (GP) equation [7] for the condensate wavefunction $\Psi(\mathbf{r}, t)$ :

$$
i \hbar \frac{\partial \Psi}{\partial t}=\left(-\frac{\hbar^{2} \nabla^{2}}{2 m}+V_{e x t}+g|\Psi|^{2}\right) \Psi .
$$

The coupling constant $g$ is proportional to the $s$-wave scattering length $a$ through $g=4 \pi \hbar^{2} a / m$. In the following we will discuss the case of repulsive interactions $(a>0)$. The anisotropic trap is represented by the confining potential $V_{e x t}$, which is chosen in the form $V_{\text {ext }}(\mathbf{r})=(m / 2) \sum_{i} \omega_{0 i}^{2} r_{i}^{2}$, where $r_{i} \equiv x, y, z$. So far the experimental traps have cylindrical symmetry and hence are characterized by the radial frequency $\omega_{\perp} \equiv$ $\omega_{x}=\omega_{y}$ and the asymmetry parameter $\lambda=\omega_{z} / \omega_{\perp}$. The ground state configuration [8,9,10] as well as the properties of small oscillations near equilibrium $11,12,13$ have been the object of systematic investigation starting from Eq. (1). A few calculations in the nonlinear regime have been also carried out 14, 15, 16, 17, 18].

In the present paper we discuss several features of the nonlinear behavior of the system, by solving Eq. (11) in the large $N$ limit, where $N=\int d \mathbf{r}|\Psi(\mathbf{r}, t)|^{2}$ is the num- ber of atoms. In this limit, it is possible to derive almost analytic results of the GP equation, thereby simplifying the numerical analysis and allowing for a systematic investigation of important phenomena. These include, the explicit time evolution of the condensate (shape of profiles, aspect ratio etc.) during the expansion and the dependence of the collective frequencies on the amplitude of the oscillation.

The effective strength of the interatomic forces in the GP equation is fixed by the adimensional parameter $N a / a_{0 i}$, where $a_{0 i}=\sqrt{\hbar /\left(m \omega_{0 i}\right)}$ is the harmonic oscillator length. When this parameter is much larger than 1 , the repulsion makes the system much wider than the noninteracting configuration, yielding a rather smooth profile. In such conditions, the equilibrium results from a balance between the external potential and the repulsive interaction, the kinetic energy playing a minor role. For large values of $N a / a_{0 i}$, one can then neglect the kinetic energy term in (1). This yields the Thomas-Fermi (TF) approximation for the ground state:

$$
\rho_{0}^{T F}(\mathbf{r})=\left|\Psi_{0}^{T F}(\mathbf{r})\right|^{2}=g^{-1}\left[\mu-V_{e x t}(\mathbf{r})\right]
$$

when $\mu>V_{\text {ext }}(\mathbf{r})$ and $\rho_{0}(\mathbf{r})=0$ elsewhere. The chemical potential $\mu$ is fixed by the normalization of the density to the number of particle $N$ :

$$
\mu=\frac{1}{2}\left[\frac{15}{4 \pi} g m^{3 / 2} \omega_{0 x} \omega_{0 y} \omega_{0 z} N\right]^{2 / 5} .
$$

The Thomas-Fermi approximation (2) works in an excellent way for the configurations realized at MIT, where $N$ is of the order of 1 million atoms and more. Conversely, in the Jila experiments of Refs. [1, 2, 3] the number of atoms is smaller $\left(10^{3}-10^{4}\right)$ and the $\mathrm{TF}$ approximation provides only a semi-quantitative description. 
Neglecting the kinetic energy pressure term in the time dependent GP equation corresponds to taking into account the effect of the kinetic energy operator in (11) only on the phase of the order parameter $\Psi$. This permits to rewrite (11) in the useful hydrodynamic form [13]:

$$
\begin{gathered}
\frac{\partial}{\partial t} \rho+\nabla \cdot(\mathbf{v} \rho)=0 \\
m \frac{\partial}{\partial t} \mathbf{v}+\nabla\left(V_{e x t}+g \rho+\frac{m v^{2}}{2}\right)=0,
\end{gathered}
$$

where density and velocity are defined by $\rho=|\Psi|^{2}$ and $\mathbf{v}=\left(\Psi^{*} \nabla \Psi-\Psi \nabla \Psi^{*}\right) \hbar /(2 m i \rho)$. Equation (我) is the usual equation of continuity, while (5) establishes the irrotational nature of the superfluid velocity. It is immediate to verify that the equilibrium configuration given by Eqs. (幽) $(\mathbf{v}=0$ and $\partial \rho / \partial t=0)$ coincides with the TF result (2).

The hydrodynamic equations (4.5) have been recently shown to provide the correct frequencies of the normal modes of the condensate in the large $N$ limit [13]. With respect to the full solution of the GP equation, which includes the effect of the kinetic energy pressure, the approach based on (4.5) has the major advantage of providing an algebraic expression for the dispersion relation of the elementary excitations. The resulting predictions quite well compare with both the Jila [2,3] and MIT [6] experiments.

For nonlinear time dependent motions, which are the object of the present work, almost analytic solutions can be found starting from equations (4.5). In fact they admit exact solutions of the form

$$
\begin{aligned}
\rho(\mathbf{r}, t) & =a_{x}(t) x^{2}+a_{y}(t) y^{2}+a_{z}(t) z^{2}+a_{0}(t) \\
\mathbf{v} & =\frac{1}{2} \nabla\left[\alpha_{x}(t) x^{2}+\alpha_{y}(t) y^{2}+\alpha_{z}(t) z^{2}\right] .
\end{aligned}
$$

Equation (6) is restricted to the region where $\rho \geq 0$ and the coefficient $a_{0}$ is fixed by the normalization of the density: $a_{0}=-(15 N / 8 \pi)^{2 / 5}\left(a_{x} a_{y} a_{z}\right)^{1 / 5}$. The time dependent coefficients $a_{i}$ and $\alpha_{i}$ obey the following coupled differential equations:

$$
\begin{aligned}
& \dot{a}_{i}+2 a_{i} \alpha_{i}+a_{i} \sum_{j} \alpha_{j}=0 \\
& \dot{\alpha}_{i}+\alpha_{i}^{2}+\omega_{0 i}^{2}+(2 g / m) a_{i}=0,
\end{aligned}
$$

with $i, j=x, y, z$. One can use the three equations (8) to express $\alpha_{i}$ in terms of $\dot{a}_{i} / a_{i}$; the solution is greatly simplified by introducing the new adimensional variables $b_{i}$ defined by $a_{i}=-m \omega_{0 i}^{2}\left(2 g b_{x} b_{y} b_{z} b_{i}^{2}\right)^{-1}$. With this choice, Eqs. (8) reduce to $\alpha_{i}=\dot{b}_{i} / b_{i}$ and Eqs. (9) become

$$
\ddot{b}_{i}+\omega_{0 i}^{2} b_{i}-\omega_{0 i}^{2} /\left(b_{i} b_{x} b_{y} b_{z}\right)=0 .
$$

The second and third terms of (10) give the effect of the external trap and of the interatomic forces, respectively.
It is worth noticing that, using the new variables $b_{i}$, the equations of motion do not depend on the value of the coupling constant $g$. This is a typical feature characterizing the large $N$ behavior of the GP equation. The mean square radii and velocities of the atomic cloud can be easily expressed in terms of $b_{i}$ :

$$
\begin{aligned}
& \left\langle r_{i}^{2}\right\rangle \equiv \frac{1}{N} \int d \mathbf{r} \rho(\mathbf{r}, t) r_{i}^{2}=\left(\frac{2 \mu}{7 m \omega_{0 i}^{2}}\right) b_{i}^{2} \\
& \left\langle v_{i}^{2}\right\rangle \equiv \frac{1}{N} \int d \mathbf{r} \rho(\mathbf{r}, t) v_{i}^{2}=\left(\frac{2 \mu}{7 m \omega_{0 i}^{2}}\right) \dot{b}_{i}^{2},
\end{aligned}
$$

where $\mu$ is given by (3).

The solutions (6-7) are well suited to describe both the oscillations around the ground state [19] and the problem of the expansion of the gas after switching off the confining potential. Equations (10) have been already derived by other authors 16, 17, using the formalism of scaling transformations, and applied to study several dynamic phenomena. In the present approach the same equations emerge as an exact solution of the hydrodynamic equations of superfluids (4-5) and will be used to investigate nonlinear oscillations and the expansion in both the Jila and MIT traps.

In order to apply the above formalism to study the expansion of the cloud, let us suppose that at $t=0$ the system is in its equilibrium configuration. Comparing Eq. (6) with the TF ground state density (2) and substituting $a_{i}$ in terms of $b_{i}$, one finds $b_{i}=1$. One has also $\dot{b}_{i}=0$ at equilibrium. The external potential is then suddenly switched off and the system starts expanding. This corresponds to solving the equations (10) with the second term set equal to zero:

$$
\ddot{b}_{i}-\omega_{0 i}^{2} /\left(b_{i} b_{x} b_{y} b_{z}\right)=0 .
$$

For an initially spherical configuration the expansion will proceed isotropically. In the presence of anisotropy, the expansion (and consequently the asymptotic velocities) will be instead faster in the direction where the repulsive forces (proportional to the gradient of the density) are stronger. For an axially deformed trap with $\lambda=\omega_{z} / \omega_{\perp} \geq 1$ this will occur in the axial direction, while if $\lambda \leq 1$ it will occur in the radial direction. The ratio

$$
R_{r}(t) \equiv \sqrt{\left\langle z^{2}\right\rangle /\left\langle x^{2}\right\rangle}=\lambda^{-1} b_{z}(t) / b_{x}(t)
$$

of the radii in the two different directions is called the aspect ratio in co-ordinate space. One can also define the aspect ratio of velocities

$$
R_{v}(t) \equiv \sqrt{\left\langle v_{z}^{2}\right\rangle /\left\langle v_{x}^{2}\right\rangle}=\lambda^{-1} \dot{b}_{z}(t) / \dot{b}_{x}(t),
$$

whose deviations from unity reflect the anysotropy of the velocity distribution. This anysotropy represents a crucial feature of Bose condensates. Asymptotically 
$(t \rightarrow \infty)$ the two aspect ratios $R_{r}$ and $R_{v}$ converge to the same value $R$. However for finite values of the expansion time they can behave quite differently. In fact, the velocities in both radial and axial directions reach their asymptotic values very rapidly, since the time scale for acceleration is very short; vice versa the radii approach their asymptotic behavior, $\propto v_{i} t$, much more slowly. This is an important feature to take into account in the analysis of experimental data.

In Figs. 1 and 2 we show the results of the two aspect ratios $R_{r}$ and $R_{v}$ obtained by solving numerically Eqs. (13) for two different sets of frequencies $\omega_{0 i}$, corresponding to the Jila [2,3] and MIT [5] traps, respectively. The different behavior of the two aspect ratios $R_{r}$ and $R_{v}$ is evident in both cases. In the same figures we have also shown the predictions of the noninteracting harmonic oscillator model, which gives $\left\langle v_{i}^{2}\right\rangle=\hbar \omega_{0 i} /(2 m)$ and $\left\langle r_{i}^{2}\right\rangle=(1 / 2) a_{0 i}^{2}+\left\langle v_{i}^{2}\right\rangle t^{2}$. The figures point out very clearly the role of two-body interactions which modify both the timescale of the expansion process and the asymptotic value of the aspect ratio. The comparison with the experimental data for $R_{r}$ should be however taken with care. In the case of the Jila experiments, $N$ is of the order of $10^{3} \div 10^{4}$, so that the TF approximation is expected to be rather crude; moreover, the points in Fig. 1 are taken from a gaussian fit to the spatial distribution of the atoms and they can be significantly corrected by using different fitting functions 115,20]. In the case of the MIT experiments, the points in Fig. 2 correspond to our estimate of the aspect ratio, extracted from the time-of-flight images in Fig. 1 of Ref. [D].

In Fig. 3 we report the asymptotic aspect ratio $R$ as a function of $\lambda$. This curve has been recently calculated also in Ref. [17]. In the limit $\lambda \rightarrow 0$ the aspect ratio approaches the value $(\pi / 2) \lambda[16$. For comparison we also show the predictions of the noninteracting harmonic oscillator model, given by $R_{H O}=\sqrt{\lambda}$.

Another important quantity to discuss is the release energy $E_{r e l}$, defined as the energy per particle of the system after the switch-off of the trap. This energy is given by the sum of the kinetic and interaction energy of the atoms and it is conserved during the expansion, being finally converted entirely into the kinetic energy of the expanding cloud. In the present formalism the release energy is a first integral of equation (13) and can be written as

$$
E_{r e l}=\frac{2 \mu}{7}\left[\frac{1}{b_{x} b_{y} b_{z}}+\frac{1}{2} \sum_{i} \frac{\dot{b}_{i}^{2}}{\omega_{0 i}^{2}}\right]=\frac{2 \mu}{7},
$$

where $\mu$ is given by (3). At the beginning of the expansion $E_{r e l}$ coincides with the 2-body interaction energy of the TF ground state. The comparison between Eq. (16) and the numerical results for $E_{k i n}+E_{i n t}$, calculated with the exact ground state solution of the Gross-Pitaevskii equation, provides a test of the validity of the TF ap- proximation. For $N \simeq 10^{3}$ the agreement with the exact result is only semiquantitative, becoming better and better as $N$ increases [9]. After long expansion time the release energy can be related to the value of the square radii of the system, through the equation

$$
E_{r e l}=\frac{m}{2}\left\langle v^{2}\right\rangle_{t \rightarrow \infty}=\frac{m}{2 t^{2}}\left\langle r^{2}\right\rangle_{t \rightarrow \infty} .
$$

The release energy has been measured both at Jila 15 and MIT [5]. It is worth noticing that in the MIT trap, where the initial configuration is a strongly anisotropic ellipsoid with the major axis along $z$, the release energy is almost entirely converted into kinetic energy of the radial motion, the velocity along $z$ being much smaller. This can be tested by solving equation (13) with the parameters appropriate for the trap of Ref. [5]. One finds that, after an expansion of $40 \mathrm{~ms}$, the 2-body interaction energy is a factor $10^{-4}$ smaller than the initial value and the ratio between the axial and radial kinetic energies is approximately $4 \times 10^{-3}$.

The same formalism can be used to investigate the oscillations of the trapped gas. One can easily check that, in the limit of small deformations, the solutions of (10) yield the dispersion relation discussed in Ref. [13]. For an axially deformed trap the normal modes are classified in terms of the third component $m$ of the angular momentum. We will discuss here the $m=0$ and $m=2$ modes which are accounted for by the parametrization (6-7). The $m=2$ mode, in the linear limit, has the frequency $\omega=\sqrt{2} \omega_{\perp}$, while the low-lying $m=0$ mode, resulting from the coupling between monopole and quadrupole oscillations, has the frequency 13] $\omega^{2}=\eta \omega_{\perp}^{2}$, with $\eta=\left(4+3 \lambda^{2}-\sqrt{9 \lambda^{4}-16 \lambda^{2}+16}\right) / 2$. The frequency of the collective modes is expected to change when the amplitude of the oscillations becomes large, due to nonlinear effects. In order to calculate such deviations, we solve (10) using, as initial conditions, the ground state values $b_{i}(0)=1$, but with $\dot{b}(0) \neq 0$. We choose the values of the velocities $\dot{b}_{i}(0)$ in order to to excite, in the linear limit, the two separate $m=2$ and $m=0$ modes. For the $m=2$ mode this implies $\dot{b}_{x}=-\dot{b}_{y}=\epsilon$ and $\dot{b}_{z}=0$, where the parameter $\epsilon$ fixes the amplitude of the oscillations. For the $m=0$ mode, one has $\dot{b}_{x}=\dot{b}_{y}=\epsilon$ and $\dot{b}_{z}=\epsilon(\eta-4)$. In this case, the system oscillates also along $z$, the axial and radial oscillations having relative amplitude $\eta-4$. It is worth noticing that the occurrence of a simultaneous oscillation in both the radial and axial widths is a typical effect of the interaction between the atoms. In fact, in the absence of 2-body forces (noninteracting harmonic oscillator), the motion in the two directions would be exactly decoupled. The experiments of Ref. [2] reveal not only a good agreement with the predicted frequencies of the two modes but also a clear evidence for the coupling between the axial and radial oscillations (see Fig. 2 of Ref. [2]). These results are crucial signatures of the important role played by the interaction. 
By increasing the initial values $\dot{b}_{i}$, it is possible to explore the nonlinear regime. A major problem for the comparison with the experimental data is that the oscillations are measured by imaging the atomic cloud after switching off the trap and leaving the atoms to expand for a few ms. During the expansion the relative amplitude of the axial and radial motions can be significantly modified. This is especially true for the MIT trap, due to the strong asymmetry of the starting cigar-shaped configuration which makes the expansion in the radial and axial directions quite different. This nontrivial evolution of the oscillating cloud is not surprising if one thinks that, after an expansion time of a few ms, the size of the system can increase by more than a factor ten. In order to make a significant comparison with the experiments it is then important to simulate both the oscillations in the trap and the subsequent expansion.

In Fig. 1 we show our predictions for the frequencies of the $m=0$ and $m=2$ modes, in the case of the Jila trap $(\lambda=\sqrt{8})$, as a function of the relative amplitude, defined as $(1 / 2)\left[\left\langle x^{2}\right\rangle_{\text {max }}^{1 / 2}-\left\langle x^{2}\right\rangle_{\text {min }}^{1 / 2}\right] /\left\langle x^{2}\right\rangle_{\text {ave }}^{1 / 2}$. The latter is calculated after expanding the trap for $7 \mathrm{~ms}$, as in the experiments of Refs. [2,3]. We find that the frequency of the $m=0$ mode does not exhibit any significant dependence on the relative amplitude, while the $m=2$ frequency increases. This agrees with the experimental findings [2, 3 , though the measured frequency shift of the $m=2$ mode is about a factor two larger than our prediction.

As already pointed out, the relative amplitude of the oscillations in the radial and axial directions behave differently during the expansion. For example, after exciting the $m=2$ mode in the trap, with a relative amplitude of $5 \%$ and $4 \%$ in the radial and axial directions respectively, the corresponding oscillations of the expanded cloud, after $7 \mathrm{~ms}$, have a relative amplitude of $9 \%$ and $4 \%$. The aspect ratio $R_{r}$ after $7 \mathrm{~ms}$ is found to be 1.65 . These predictions rather well agree with the experiments (see Fig. 2 of Ref. [2]), where the aspect ratio is found to be 1.75 and the radial and axial relative amplitudes are $10 \%$ and $4 \%$, respectively.

We have repeated the same calculations for the cigarshaped trap of the MIT experiments $(\lambda=0.077$ [6]). In this case we excite the low-lying $m=0$ mode in the trap and then we calculate the frequency and the amplitude of the oscillations after expanding the gas for $40 \mathrm{~ms}$. We do not observe any frequency shift of the low-lying $m=0$ mode as a function of the amplitude, in agreement with the experiments. One should note that the nonlinearity of the oscillations are amplified during the expansion much more than in the Jila trap, since the system is very anisotropic. As a consequence, the large axial amplitude observed after an expansion of $40 \mathrm{~ms}$ corresponds to a rather small amplitude at $t=0$ and this explain in part the absence of shift in the frequency. As an example, let us consider the aspect ratio plotted in Fig. 2 of
Ref. [6], which oscillates between about 0.28 and 0.38 . One can easily reproduce the same oscillations by solving (13) starting from a configuration which oscillates as a low-lying $m=0$ mode in the trap (see also Ref. [16]). To get the measured aspect ratio one has to start with an oscillation having relative amplitude less than $1 \%$ in the radial direction and about $3.3 \%$ in the axial one. During the expansion the relative amplitude of the radial motion remains practically unchanged while the one of the axial motion increases up to $15 \%$. These large fluctuations of the axial width of the system are compatible with the conservation of energy, since almost all the energy is carried by the motion of the atoms in the radial expansion, which is much faster than the axial one.

It is also worth noticing that, when $\lambda \ll 1$, the frequency of the high-lying $m=0$ mode is exactly $2 \omega_{\perp}$ even in the nonlinear regime. In this limit, this mode corresponds to a two-dimensional motion and the absence of a shift in its frequency reflects the occurrence of a hidden symmetry of a 2D Bose gas in a harmonic trap [21].

In conclusion we have shown that the hydrodynamic equations of superfluids provide a useful description of several nonlinear effects, associated with the dynamic behavior of a trapped Bose gas at zero temperature. A reasonable agreement with the first available experimental data is found, though for a more quantitative comparison, when the number of atoms is relatively small, the complete solution of the Gross-Pitaevskii equations is expected to be relevant. Our analysis points out the crucial role played by the interatomic forces in the dynamics of the expansion as well as in the behavior of the collective excitations. A natural extension of this work should include thermal effects and, in particular, the interaction between the consensed and thermal components of these systems.

[1] M. H. Anderson, J. R. Ensher, M. R. Matthews, C. E. Wieman, and E. A. Cornell, Science 269, 198 (1995).

[2] D. S. Jin, J. R. Ensher, M. R. Matthews, C. E. Wiemann, and E. A. Cornell, Phys. Rev. Lett. 77, 420 (1996).

[3] D. S. Jin, M. R. Matthews, J. R. Ensher, C. E. Wiemann, and E. A. Cornell, preprint 1996

[4] K. B. Davis, M.-O. Mewes, M. R. Andrews, N. J. van Druten, D. S. Durfee, D. M. Kurn, and W. Ketterle, Phys. Rev. Lett. 75, 3969 (1995).

[5] M.-O. Mewes, M. R. Andrews, N. J. van Druten, D. M. Kurn, D. S. Durfee, and W. Ketterle, Phys. Rev. Lett. 77, 416 (1996)

[6] M.-O. Mewes, M. R. Andrews, N. J. van Druten, D. M. Kurn, D. S. Durfee, C. G. Townsend, and W. Ketterle, Phys. Rev. Lett. 77, 988 (1996)

[7] L.P. Pitaevskii, Zh. Eksp. Teor. Fiz. 40, 646 (1961) [Sov. 
Phys. JETP 13, 451 (1961)]; E.P. Gross, Nuovo Cimento 20, 454 (1961); E.P. Gross, J. Math. Phys. 4, 195 (1963).

[8] G. Baym and C. Pethick, Phys. Rev. Lett. 76, 6 (1996).

[9] F. Dalfovo and S. Stringari, Phys. Rev. A 53, 4377 (1996).

[10] Mark Edwards, R. J. Dodd, C. W. Clark, P. A. Ruprecht, and K. Burnett, Phys. Rev. A 53, R1950 (1996).

[11] Mark Edwards, P. A. Ruprecht, K. Burnett, R. J. Dodd, and C. W. Clark, Phys. Rev. Lett. 77, 1671 (1996).

[12] K.G. Singh and D.S. Rokhsar, Phys. Rev. Lett. 77, 1667 (1996)

[13] S. Stringari, Phys. Rev. Lett. 77, 2360 (1996)

[14] M. J. Holland and J. Cooper, Phys. Rev. A 53, R1954 (1996).

[15] M.J. Holland, D. Jin, M.L. Chiofalo, and J. Cooper, preprint 1996

[16] Y. Castin and R. Dum, preprint 1996

[17] Yu. Kagan, E.L. Surkov and G.V. Shlyapnikov, Phys. rev. A 54, R1753 (1996); Yu. Kagan, E.L. Surkov and G.V. Shlyapnikov, preprint

[18] P.A. Ruprecht, M. Edwards, K. Burnett, and C.W. Clark, Phys. Rev. A 54, 4178 (1996)

[19] Another exact solution of Eqs. (45) is obtained including terms linear in $x$ (or $y, z$ ) in the density and in the velocity potential. This solution corresponds to the motion of the center of mass of the cloud. Other solutions can be found including terms of the form $x y(x z, y z)$.

[20] D. S. Jin and E. A. Cornell, private communication

[21] L.P. Pitaevskii, Phys. Lett. A 221, 14 (1996); L.P. Pitaevskii and A. Rosch, preprint cond-mat/9608135

FIG. 1. Aspect ratio for the Jila trap $\left(\omega_{\perp}=2 \pi 132 \mathrm{~Hz}\right.$ and $\lambda=\sqrt{8})$ as a function of the expansion time. Upper and lower solid lines correspond to $R_{v}$ and $R_{r}$, respectively. Dashed lines are the same quantity for the noninteracting particles. Points are experimental values of $R_{r}$ taken from 20.

FIG. 2. Aspect ratio for the MIT trap $\left(\omega_{\perp}=2 \pi 320 \mathrm{~Hz}\right.$ and $\lambda=0.056)$ as a function of the expansion time. Lower and upper solid lines correspond to $R_{v}$ and $R_{r}$, respectively. Dashed lines are the same quantity for the noninteracting particles. Points are experimental values of $R_{r}$ extracted from Ref. [5] (see text).

FIG. 3. Asymptotic aspect ratio vs. $\lambda=\omega_{z} / \omega_{\perp}$. Solid lines: from (13); dashed line: noninteracting gas.

FIG. 4. Frequencies of the collective modes as a function of the relative radial amplitude, for the $m=0$ (triangles) and $m=2$ (circles) modes in the Jila trap. Solid lines: from (10); points: from Refs. [3, 阳]. 


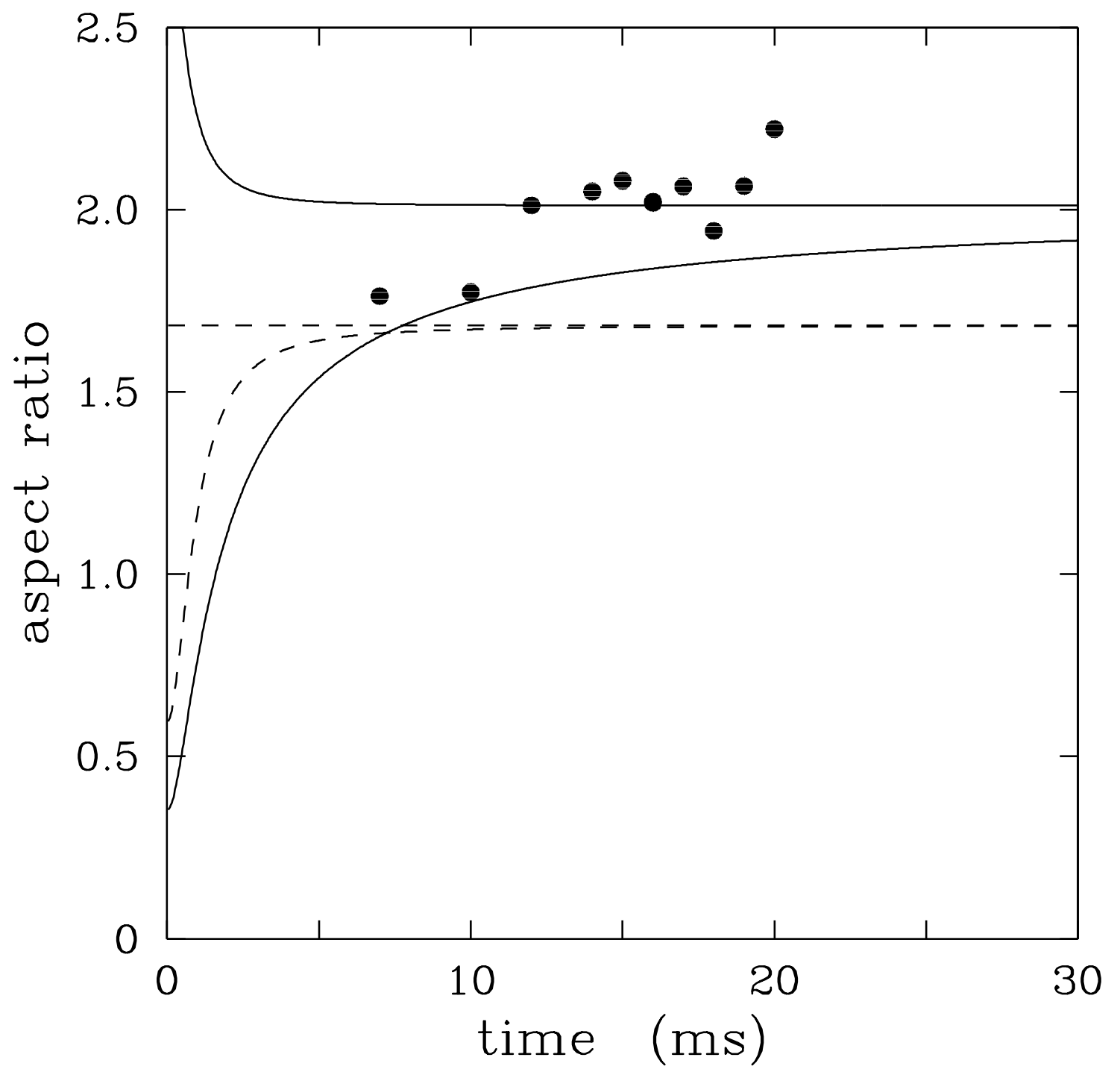




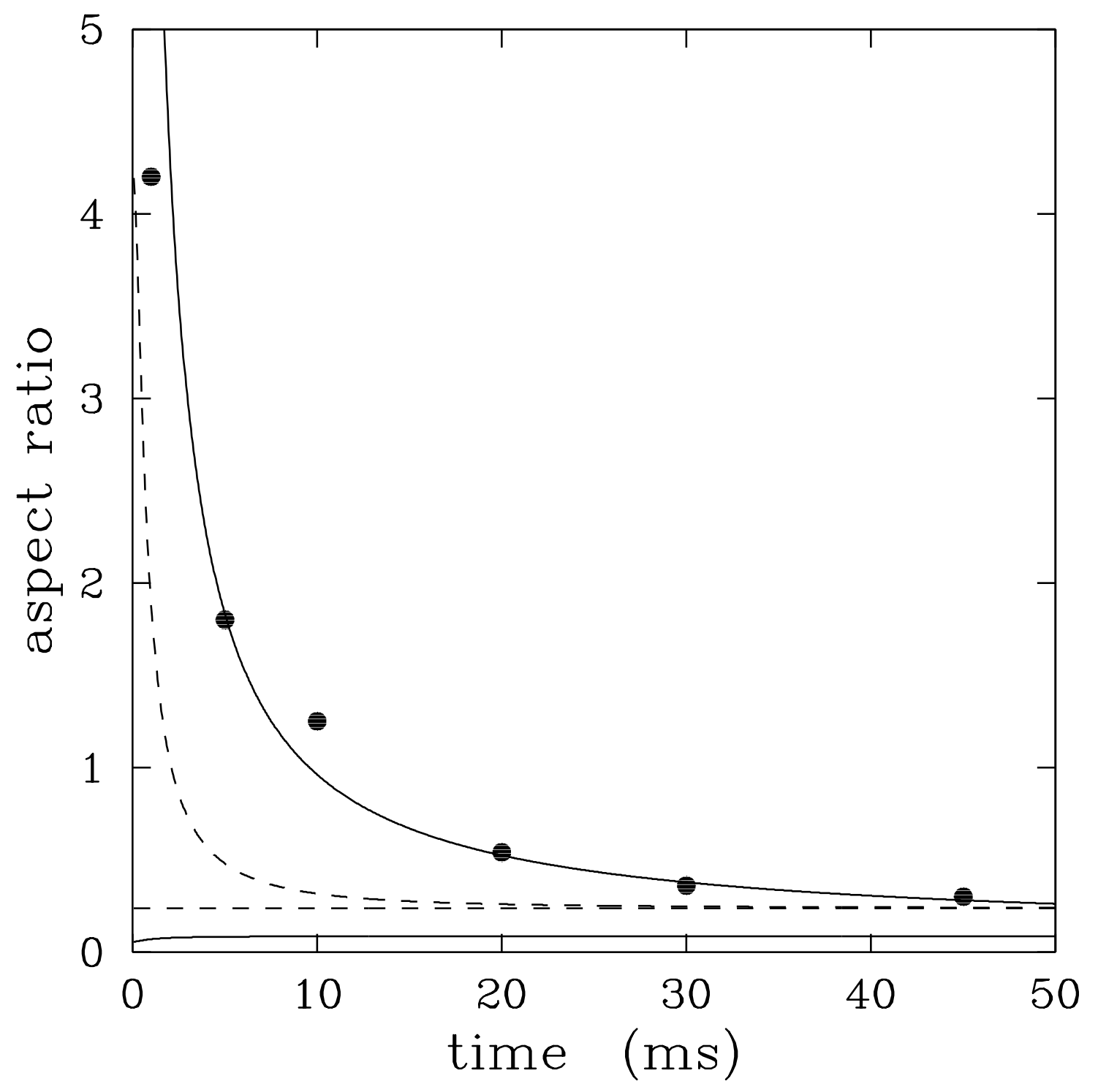











